\title{
The ARSQ: The Athletes' Received Support Questionnaire
}

2

3 Please reference this paper as:

4 Freeman, P., Coffee, P., Moll, T., Rees, T., \& Sammy, N. (2014). The ARSQ: The Athletes' Received Support Questionnaire. Journal of Sport \& Exercise Psychology, 36(2), 189-202. doi:10.1123/jsep.2013-0080 
Abstract

3 To address calls for context-specific measurement of social support, this article reports the

4 development of the Athletes' Received Support Questionnaire (ARSQ) and demonstrates initial

5 evidence for its validity. Across four studies there was support for a four-dimensional structure

6 reflecting emotional, esteem, informational, and tangible received support. There was also

7 support for unidimensional and higher-order models. Further, Study 3 provided support for

8 convergent validity, with significant correlations between the corresponding dimensions of the

9 ARSQ and the Inventory of Socially Supportive Behaviors. Study 4 provided evidence for the

10 nomological validity of the ARSQ. Emotional and esteem support significantly predicted self-

11 confidence and positive affect, and tangible support significantly moderated the relationship

12 between stress and negative affect. Collectively, these results provide evidence for the validity

13 of the ARSQ, and offer researchers flexibility to adopt either a multidimensional or aggregated

14 approach to measuring received support.

15 Keywords: partial least squares structural equation modeling, social support, sport

16 psychology 
Social support has become increasingly recognized as a key resource for athletes. For

3 example, studies employing qualitative methods have found links between social support and

4 enhanced coping with organizational stressors (Kristiansen, Murphy, \& Roberts, 2012), return

5 from injury (Carson \& Polman, 2012), and psychological resilience in Olympic champions

6 (Fletcher \& Sarkar, 2012). Studies employing quantitative methods have found links between

7 social support and self-confidence (Rees \& Freeman, 2007), athletes' self-talk (Zourbanos et al.,

8 2011), and lower levels of burnout (Raedeke \& Smith, 2004). With this increasing interest, there

9 has been greater diversity in the conceptualization and measurement of social support, making

10 synthesis of findings difficult. To enhance understanding of the impact of social support, it is

11 vital that researchers use theoretically-based measures with sound psychometric properties. The

12 purpose of the present study was to develop a measure of received support and provide initial

13 evidence of its validity.

14 In both sport and social psychology, social support has been recognized as multi-faceted,

15 comprising structural and functional components (Cohen, Gottlieb, \& Underwood, 2000; Holt \&

16 Hoar, 2006; Rees, 2007; Vangelisti, 2009). Structural components are the type and number of

17 relationships in which an individual engages and whether one is integrated within social groups.

18 Functional components are the functions served by interpersonal relationships, and are further

19 divided into perceived and received support, which have both been assessed in terms of quantity

20 and satisfaction (for reviews, see Cohen et al., 2000; Holt \& Hoar, 2006). Perceived support

21 typically refers to an individual's belief that assistance would be available if required. Received

22 support typically refers to the frequency with which an individual has received supportive

23 resources during a specific time frame and is usually assessed with retrospective self-reports 
1 (Gottlieb \& Bergen, 2010). Perceived and received support typically share around 12\% common

2 variance (Haber, Cohen, Lucas, \& Baltes, 2007) and can exert unique effects upon outcomes

3 such as sports performance (Freeman \& Rees, 2008). As such, it is imperative that sport

4 psychology researchers are clear in how they conceptualize and measure social support (Bianco

$5 \quad \&$ Eklund, 2001; Holt \& Hoar, 2006).

6 A range of measures have been used to assess social support in sport, including ones that

7 were originally developed in social psychology, such as the Social Support Questionnaire

8 (Sarason, Levine, Basham, \& Sarason, 1983), the Social Support Survey (Richman, Rosenfeld,

9 \& Hardy, 1993), and the Interpersonal Support Evaluation List (Cohen, Mermelstein, Kamarck,

$10 \&$ Hoberman, 1985). The use of such measures in sport has been questioned, however, because

11 they do not necessarily reflect the specific forms of support that are required by athletes (Rees,

12 Ingledew, \& Hardy, 1999). For example, Rees and Hardy (2000) found that athletes received

13 unique forms of support for sport-specific demands such as dealing with injuries, pre-

14 competition nerves, performance catastrophes, and technical difficulties, in addition to receiving

15 support regarding everyday issues. As such, there have been calls to develop measures of social

16 support that are relevant to the support experiences of athletes (Bianco \& Eklund, 2001; Holt \&

17 Hoar, 2006; Rees, 2007).

18 One way these calls have been addressed is through the creation of context-specific

19 measures for particular studies. By using this approach, researchers (e.g., Freeman \& Rees,

20 2008; Lubans, Morgan, \& McCormack, 2011; Zourbanos et al., 2011) have ensured their

21 measures have good content validity. In addition, such studies have often demonstrated notable

22 measurement sophistication. This has enriched understanding into received support by

23 demonstrating links to golf performance (Freeman \& Rees, 2008), adolescents' beliefs about 
1 school sport (Lubans et al., 2011), and athletes' self-talk (Zourbanos et al., 2011). It has been

2 argued, however, that creating unique measures of support for particular studies hinders the 3 comparison of results across the literature (Holt \& Hoar, 2006; Vangelisti, 2009). For example,

4 it is unclear whether the observed relationships are due to the theoretical support constructs or

5 influenced by the idiosyncratic properties of the unique measures, such as their specific items or

6 subscales. Further, the trend to create measures for particular studies does not encourage social

7 support research, because the development and psychometric testing of measures is a time-

8 consuming process. To overcome these concerns, the Perceived Available Support in Sport

9 Questionnaire (PASS-Q; Freeman, Coffee, \& Rees, 2011) was recently designed to be used

10 across all sporting contexts to assess perceived support, but no equivalent measure exists for

11 received support.

12 The development and consistent use of a received support measure could help address a

13 number of important issues, including the extent to which received support is beneficial, how

14 received support operates, and factors that moderate its effectiveness. As noted above, empirical

15 evidence in sport has demonstrated that received support is linked with favorable outcomes, but

16 some studies in both sport (e.g., Udry, Gould, Bridges, \& Tuffey, 1997) and social (e.g.,

17 Reinhardt, Boerner, \& Horowitz, 2006) psychology have found that not all supportive attempts

18 are beneficial. Theory and empirical evidence has highlighted that there are different models

19 which may explain how received support influences outcomes (for reviews, see Bianco \&

20 Eklund, 2001; Cohen et al., 2000). Bianco and Eklund (2001) argued that perceived support is

21 primarily linked with the main effect model, and received support is primarily linked with stress-

22 buffering effect model. Other researchers have, however, suggested that both perceived and

23 received support could exert main and stress-buffering effects (Cohen et al., 2000). The stress- 
1 buffering model suggests that social support moderates the relationship between stress and

2 outcomes: at low levels of support, stress is negatively related to outcomes, but at high levels of

3 support stress is unrelated to outcomes (Cohen et al., 2000). The main effect model suggests that

4 social support is directly related to outcomes irrespective of levels of stress. Identifying the

5 conditions under which received support is beneficial will advance theory and the development

6 of theory-led support interventions. For example, evidence for stress-buffering effects would

7 imply that received support is primarily beneficial only when athletes are under stress.

8 An important issue to consider in the development of a measure is whether received

9 support should be conceptualized as a unidimensional or multidimensional construct. Some

10 researchers have employed an overall score of received support (e.g., Freeman \& Rees, 2008).

11 As different supportive behaviors have been shown to have unique effects on outcomes (e.g.,

12 Barry, Bunde, Brock, \& Lawrence, 2009; Reinhardt et al., 2006) a multidimensional approach to

13 conceptualizing received support is, however, likely to be most appropriate. A number of

14 multidimensional models of social support have been proposed, but there is consensus across

15 both the social (e.g., Cutrona \& Russell, 1990) and sport (e.g., Rees \& Hardy, 2000) psychology

16 literatures that there are four dimensions within functional types of support: emotional, esteem,

17 informational, and tangible support. Emotional support refers to the provision of comfort,

18 security, and a sense that an individual is loved and cared for. Esteem support refers to the

19 bolstering of an individual's sense of competence. Informational support refers to the provision

20 of advice and guidance. Tangible support refers to the provision of practical and instrumental

21 assistance. A multidimensional measure could help future studies in sport quantify the direction

22 and magnitude of relationships between these received support dimensions and outcomes.

23 Across four studies, the present article reports the development of a received support 
1 questionnaire for athletes (Athletes' Received Support Questionnaire: ARSQ) and provides

2 evidence of its validity. The ARSQ is designed to be used across all sports and assesses the

3 frequency with which an athlete has received emotional, esteem, informational, and tangible

4 support over the last week. One week was chosen for two reasons: 1) because of the regularity

5 with which many athletes train and compete in their sport, and 2) to minimize the recall period.

6 After initial construction of the ARSQ, Study 1 examined its content validity. Study 2 examined

7 the proposed dimensional structure of the ARSQ. Study 3 confirmed the dimensional structure

8 with a separate sample of athletes, examined its convergent validity by testing correlations

9 between the dimensions in the ARSQ and the Inventory of Socially Supportive Behaviors (ISSB:

10 Barrera, Sandler, \& Ramsey, 1981), and tested if scores on the ARSQ were unrelated to negative

11 affectivity and social desirability. Study 4 further confirmed the dimensional structure and

12 examined the nomological validity of the ARSQ. Theoretically, received support can influence a

13 range of emotional, behavioral, cognitive, and physiological outcomes (Cohen et al., 2000). For

14 example, in sport received support has been linked with self-confidence (Rees \& Freeman,

15 2007), and in social psychology received support has been linked with affective states (e.g.,

16 Lakey, Orehek, Hain, \& Van Vleet, 2010). Therefore, we examined main and stress-buffering

17 effects of the ARSQ dimensions on self-confidence and affect.

18 We hypothesized that a four-dimensional model would demonstrate a good model fit.

19 We also hypothesized that the correlations between the ARSQ dimensions and their

20 corresponding ISSB dimensions would be significant, the remaining correlations between the

21 ARSQ and ISSB dimensions would be of lower magnitude, and there would be no significant

22 correlations between the ARSQ and either negative affectivity or social desirability. We

23 hypothesized that the ARSQ dimensions would be associated with main and stress-buffering 
1 effects on self-confidence, positive affect, and negative affect. Main effects would show that

2 higher levels of received support predict higher self-confidence and positive affect, and lower

3 negative affect. Stress-buffering effects would show that there are detrimental relationships

4 between stress and self-confidence, positive affect, and negative affect at low levels of received

5 support, but these relationships are weaker at high levels of received support.

\section{Study 1}

\section{$7 \quad$ Initial Scale Construction}

To generate items for the ARSQ we primarily referred to statements made by athletes

9 about their social support experiences (Rees \& Hardy, 2000), but also examined previous

10 received support measures designed for specific studies in sporting contexts (Freeman \& Rees,

11 2008; Zourbanos et al., 2011) and two more established social support questionnaires: the PASS-

12 Q and the ISSB. Although the PASS-Q and ISSB differ from the ARSQ in terms of type of

13 support and context respectively, some of their specific items are relevant to the support that

14 athletes receive. Initially, two authors identified 91 items for discussion. The items were then

15 reviewed for wording, redundancy, their relevance for athletes competing across a range of

16 sports and competitive levels, and fit within either received emotional, esteem, informational, or

17 tangible support. This reduced the pool to 31 items, which were then reviewed by two further

18 authors. Nine items were removed due to debate over their relevance across all sports and

19 competitive levels or which dimension they belonged to. A final 22 items were identified for

20 inclusion in the ARSQ. The emotional and esteem support dimensions both consisted of five

21 items, and the informational and tangible support dimensions consisted of six items.

\section{Participants}

23 The 22 items were assessed by 41 sport and exercise science students (16 females, 25 
1 males; $M_{\text {age }} 21.5$ years, $S D=0.7$ ), who had all completed modules detailing social support theory

2 and research methods. The students competed in various team $(n=28)$ and individual $(n=13)$

3 sports at club $(n=20)$, regional $(n=11)$, national $(n=7)$, or international $(n=3)$ level.

\section{Procedures}

5 A university ethics committee granted ethical approval, and participants provided

6 informed consent. Participants completed the measures in a lecture theatre. Participation was

7 voluntary, with no course credits or financial incentives offered.

\section{Measures}

9 Participants were provided with definitions of the four dimensions of support, asked to 10 read each item and then write which dimension the item belonged to (Dunn, Bouffard, \& Rogers, 11 1999). Participants then rated how well they understood each item (0-4; not at all well -

12 extremely well) and its relevance to athletes across a range of sports and competitive levels (0-4;

13 not at all relevant - extremely relevant).

\section{Analyses}

15 The percentage of participants who correctly assigned each item to its dimension was

16 calculated. Item content validity indices for both understanding and relevance were calculated as

17 the proportion of participants who responded with a 3 or 4 (Polit \& Beck, 2006). A scale content

18 validity index was calculated for understanding and relevance as the mean of their respective

19 item content validity indices (Polit \& Beck, 2006).

\section{Results and Discussion}

21 All items were correctly assigned to their dimension by at least $75 \%$ of students, well

22 understood $(M \mathrm{~s}=3.02-3.90, S D \mathrm{~s}=0.30-1.05)$, and deemed to be relevant $(M \mathrm{~s}=2.65-3.78, S D \mathrm{~s}$

$23=0.42-1.05)$. The item content validity indices were $.78-1.00$ for both understanding and 
1 relevance. The scale content validity index was .94 for understanding and .92 for relevance.

2 These values exceed the thresholds suggested by Polit and Beck (2006) and provided initial

3 evidence for the content validity of the measure. Study 2 explored the dimensional structure of

4 the 22-item ARSQ.

5

6

$7 \quad$ Participants

11 did not report their ethnicity). Participants had competed for a mean of 9.1 years $(S D=5.1)$ in

12 various individual $(n=101)$ and team $(n=192)$ sports at recreational $(n=30)$, club $(n=143)$,

13 regional $(n=76)$, national $(n=33)$, or international $(n=11)$ level.

\section{Procedures}

16 informed consent. Participants completed the ARSQ in a lecture theatre. Participation was

17 voluntary with no course credits or financial incentives offered.

\section{Measures}

\section{Study 2}

\section{Method}




\section{Analyses}

A key consideration when analyzing a measurement model is whether constructs should

3 be conceptualized as reflective or formative (MacKenzie, Podsakoff, \& Podsakoff, 2011).

4 Constructs themselves, however, are not inherently reflective or formative; how they are

5 conceptualized in a study should guide the measurement approach (Diamantopoulos \&

6 Winklhofer, 2001; MacKenzie et al., 2011). To distinguish between reflective and formative

7 approaches, researchers should consider the conceptual relationship between the items and

8 construct, and the expected intercorrelations between items (Diamantopoulos \& Winklhofer,

9 2001). Reflective models propose that the items reflect (are caused by) a latent variable; because

10 items share this conceptual unity, they should have high intercorrelations. In contrast, formative

11 models propose that the items form a composite variable, and no a priori assumption is made

12 regarding the intercorrelations between items. In our opinion, received support measures,

13 particularly those with frequency style response options, may be best characterized by a

14 formative approach. Although received support may primarily arise in the context of established

15 relationships, the reported frequencies of supportive behaviors are not a reflection of a common

16 latent construct, but rather a recall of the number of incidents of support. The receipt of these

17 behaviors defines the level of received support, and the receipt of one supportive behavior does

18 not necessarily mean other behaviors will also be received (Gottlieb \& Bergen, 2010).

19 In line with a formative approach, in this article we used partial least squares structural

20 equation modeling, which can handle formative and/or reflective measures, and is able to deal

21 with small sample sizes and non-normality (Hair, Sarstedt, Ringle, \& Mena, 2012). We

22 employed WarpPLS 3.0 (Kock, 2012) with the Warp3 PLS regression algorithm and

23 bootstrapping with 100 resamples to estimate probability values for significance testing. 
Initially, a four-dimensional model was examined comprising emotional, esteem,

2 informational, and tangible support. Evidence for the discriminant validity of the dimensions

3 was provided if the $95 \%$ confidence intervals around the correlations between dimensions did

4 not contain 1. We also examined alternative dimensional structures: a unidimensional model and

5 a four-dimensional model with a single higher-order construct. Received support has been

6 suggested to be unidimensional and researchers have adopted a composite score when examining

7 the effects of received support (e.g., Freeman \& Rees, 2008). Further, Holt and Hoar (2006)

8 noted that although a distinction can be made between support dimensions, they might also be

9 subsumed within a single higher-order construct reflecting global received support. In the

10 higher-order model, the four first-order dimension scores were treated as formative indicators of

11 a higher-order composite received support construct.

12 Given the formative approach adopted, emphasis was placed on the indicator (item)

13 weights and their statistical significance rather than traditional indices of model fit used in

14 reflective confirmatory factor analysis (Hair et al., 2012). These indicator weights are

15 standardized multiple regression coefficients, and each composite variable (e.g., received support

16 dimension) was formed by the linear combination of its indicators (items). Evidence for item

17 validity was provided if the indicator weight was significant $(p<.05$; Kock, 2012). We also

18 examined the variance inflation factors (VIFs) of the indicators and full collinearity VIFs for the

19 composite variables. These were used to assess item and dimension redundancy. Indicator VIFs

20 assess the extent to which items measure the same aspect of a composite variable. Full

21 collinearity VIFs assess the extent of collinearity between composite variables. Although

22 various thresholds for VIFs have been suggested in the multivariate analysis literature,

23 researchers (e.g., Diamantopoulos \& Winklhofer, 2001; MacKenzie et al., 2011) have suggested 
1 that VIFs greater than 10 signify items and dimensions could be considered for elimination.

\section{Results and Discussion}

\section{Four-Dimensional Model}

$4 \quad$ For the four-dimensional model, all indicator weights were significant (see Table 1)

5 suggesting that each item made a significant contribution to its respective dimension. The mean

6 of the indicator VIFs was 2.45 (range 1.56-3.24), suggesting that there were no collinearity

7 concerns (Diamantopoulos \& Winklhofer, 2001; MacKenzie et al., 2011) and that the items

8 measured different facets of their respective support dimension. The mean of the full collinearity

9 VIFs for the four dimensions was 3.36, suggesting that the dimensions measured different facets

10 of received support. The $95 \%$ confidence intervals around the correlations between dimensions

11 ranged from .48 to .86 providing some further evidence for discriminant validity of the

12 dimensions. The correlations, however, were quite high ( $p$ s $<.001)$, ranging from $r=.57$

13 (emotional and tangible) to .82 (esteem and informational). Although received support may be

14 conceptually divided into distinct dimensions, in naturalistic settings the correlations between

15 them are often significant (Finch et al., 1997; Rees, Hardy, \& Freeman, 2007), because those

16 people who support athletes can do so in multiple ways. For example, a coach might offer

17 encouragement alongside technical advice. Therefore, it was important to explore whether

18 alternative models could account for the correlations between dimensions.

\section{Alternative models}

For the unidimensional model, all indicator weights were significant $(b \mathrm{~s}=.05-.07, S E \mathrm{~s}=$

$21.01, p \mathrm{~s}<.001$ ). The mean of the indicator VIFs was 2.94 (range 1.93-3.80). As such, there was

22 support for a unidimensional model. For the higher-order model, all four indicator weights from

23 the support dimensions to the high-order construct were significant $(b \mathrm{~s}=.27-.30, S E \mathbf{s}=.01-.02$, 
$1 p \mathrm{~s}<.001)$ and the indicator VIFs were 2.43-4.25 $(M=3.36)$. The results for the additional

2 models, therefore, suggest that adopting a unidimensional or higher-order support construct may

3 be appropriate. Indeed, these models would offer a more parsimonious approach than a four-

4 dimensional model. Further, the presence of a higher-order construct offers researchers the

5 potential to measure received support at a global or dimensional level. Overall the findings of

6 Study 2 provide insight into the factorial validity of the 22-item ARSQ, but further examination

7 of the dimensional structure and other psychometric properties is warranted to reveal if the

8 findings are robust across different samples and if the ARSQ satisfies other forms of validity.

Study 3

\section{Method}

\section{Participants}

12 Participants were $306\left(122\right.$ female, 184 male; $M_{\text {age }} 19.5$ years, $\left.S D=2.3\right)$ athletes who

13 competed in the British Universities and Colleges Sport competitions. The sample was

14 predominantly White British (90.2\%); no other ethnicity accounted for more than $2 \%$ of the

15 sample (3 participants did not report their ethnicity). Participants had competed for a mean of

169.4 years $(S D=4.4)$ in various individual $(n=95)$ and team $(n=211)$ sports.

\section{Procedures}

18 A university ethics committee granted ethical approval and participants provided

19 informed consent. Convenience sampling was used to recruit participants at training sessions.

20 Participants completed measures of received support, negative affectivity, and social desirability.

\section{Measures}

ARSQ. The 22 items from Study 2 were used. No modifications were made to the

23 items, the generic stem that preceded items, or the response options. 
Inventory of Socially Supportive Behaviors. To examine the convergent validity of the

2 ARSQ, participants also completed the ISSB (Barrera et al., 1981). The ISSB assesses the

3 frequency with which an individual has received support, and is the most widely used measure of

4 received support in social psychology, with evidence of good reliability and validity (Gottlieb \&

5 Bergen, 2010). In the present study, participants completed the 34-item version of the ISSB

6 validated by Finch et al. (1997), which assesses nondirective support (5 items), positive social

7 exchange (6 items), directive guidance (13 items), and tangible assistance (10 items). In the

8 present study, items were treated as formative indicators of their respective dimensions. The

9 ISSB uses different terminology than the ARSQ to label dimensions, but nondirective support

10 reflects emotional support, positive social exchange reflects esteem support, directive guidance

11 reflects informational support, and tangible assistance reflects tangible support. Participants

12 rated the frequency with which they had received supportive behaviors in the last month on a

13 five-point scale: not at all, once or twice, about once a week, several times a week, and about

14 every day (coded 0-4 for analysis).

15 Negative Affectivity. Negative affectivity was assessed using the Type D Scale-14

16 (Denollet, 2005). Denollet demonstrated that the negative affectivity scale was internally

17 consistent, had good test-retest reliability, and was not related to mood or health status.

18 Participants rated the seven statements on a five-point scale ranging from 0 (false) to 4 (true).

19 The reflective latent variable score was calculated, with higher scores reflecting higher levels of

20 negative affectivity. The coefficient alpha reliability was .86 in the present study.

Social Desirability. Participants completed the 13-item version of the Marlowe-Crowne

22 Social Desirability Scale (Reynolds, 1982), which Reynolds found had good internal consistency

23 and was highly correlated with the 33-item version of the scale. Participants rated whether 13 
1 statements concerning personal attitudes and traits were true (coded 1) or false (coded 0).

2 Negatively phrased items were reverse scored so that higher scores reflected socially desirable

3 attitudes and the reflective latent variable score was calculated.

4 Analyses

We first examined the four-dimensional, unidimensional, and higher-order models of the

6 ARSQ. Correlations between the four ARSQ dimensions, negative affectivity, social

7 desirability, and the corresponding ISSB dimensions were calculated using WarpPLS 3.0. A $\chi^{2}$

8 analysis was used to examine the proportion of the 12 remaining correlations between ARSQ and

9 ISSB dimensions above and below the mean of the four hypothesized correlations. As the

10 assumption of independence was violated in the $\chi^{2}$ analysis, bootstrapping with 100 resamples

11 was employed to create a $95 \%$ confidence interval, which was used to determine statistical

12 significance. An alpha level of .05 was used for all statistical tests.

\section{Results and Discussion}

14 The results replicated those found in Study 2. For the four-dimensional model, all

15 indicator weights were significant (see Table 1) and the mean of the indicator VIFs was 1.80

16 (range 1.23-2.26). The correlations between the four dimensions were moderate to high ( $p$ s $<$

17.001 ), ranging from $r=.41$ (emotional and tangible) to .71 (informational and tangible). Despite

18 these correlations, the mean of the full collinearity VIFs for the four dimensions was 2.60

19 providing some evidence that the subscales are sufficiently distinct to warrant being treated as

20 separate dimensions.

21 The unidimensional and higher-order models were also good. For the unidimensional

22 model, all indicator weights were significant $(b \mathrm{~s}=.04-.08, S E \mathrm{~s}=.01, p \mathrm{~s}<.001)$ and the mean of

23 the indicator VIFs was 2.10 (range 1.33-2.54). For the higher-order model, all four indicator 
1 weights to the higher-order construct were significant $(b \mathrm{~s}=.27-.32, S E \mathbf{s}=.01, p \mathrm{~s}<.001)$, and

2 the indicator VIFs were 2.01-3.13 $(M=2.60)$. These findings provide further evidence, with an

3 independent sample, that the dimensional structure of the ARSQ could be conceptualized in

4 different ways. This provides promising flexibility for researchers depending on the goals of

5 their studies. Across both Studies 2 and 3, there was evidence that the four dimensions can, if

6 desired, be conceptualized as distinct. A multidimensional approach offers potential to reveal

7 unique effects for the different support dimensions. Alternatively, the unidimensional and

8 higher-order models offer a more parsimonious approach, if researchers are interested in the

9 overall effects of received support.

The ARSQ dimensions were not significantly correlated with negative affectivity $(r \mathrm{~s}=$

$11.01-.11, p s=.05-.93)$. The correlations between the ARSQ dimensions and social desirability

12 were $r \mathrm{~s}=-.05(p=.42)$ to $-.13(p=.03)$. Only emotional support was significantly correlated

13 with social desirability and the magnitude of this correlation was low and indicated that higher

14 levels of emotional support were actually related to less socially desirable attitudes.

15 The correlations between the corresponding dimensions of the ARSQ and ISSB were all

16 significant $(r \mathrm{~s} .39-.56, \mathrm{ps}<.001)$; the mean value of these four correlations was $r=.50$. The

17 mean of the 12 correlations between the non-corresponding dimensions of the ARSQ and ISSB

18 was similar $(r=.42)$, raising some concerns over the convergent and discriminant validity of the

19 dimensions. Only two of the correlations between the non-corresponding ARSQ and ISSB

20 dimensions, however, were greater than .50 , with 10 of the correlations less than $.50(r \mathrm{~s}=.28$ -

$21.54, p \mathrm{~s}<.001)$; a $\chi^{2}$ analysis demonstrated that the proportion of these correlations that were

22 below .50 was significantly different than might be expected due to chance, $\chi^{2}(1)=5.34,95 \%$

23 CI $[0.12,10.56]$. These findings provide partial evidence for the convergent validity of the 
1 ARSQ, but examination of the nomological validity is warranted.

\section{Study 4}

\section{Method}

\section{$4 \quad$ Participants}

Participants were 219 (126 female, 93 male; $M_{\text {age }} 22.2$ years, $S D=5.3$ ) competitive

6 athletes. The sample was predominantly White British (87.2\%); no other ethnic group accounted

7 for more than $5 \%$ of the sample (2 participants did not report their ethnicity). Participants had

8 competed for a mean of 9.3 years $(S D=6.0)$ in various individual $(n=47)$ and team $(n=172)$

9 sports at club $(n=118)$, regional $(n=63)$, national $(n=28)$, or international $(n=10)$ level.

\section{Procedures}

11 A university ethics committee granted ethical approval and participants provided

12 informed consent. Convenience sampling was used with participants recruited at training

13 sessions. Data were collected at two time points. One week before a competition, participants

14 completed measures of stress, self-confidence, positive affect, and negative affect in relation to

15 the competition. One day before the same competition, participants completed the ARSQ, and

16 measures of self-confidence, positive affect, and negative affect in relation to the competition.

\section{Measures}

ARSQ. The 22 items from Studies 1-3 were used. No modifications were made.

Stress. Stress was assessed using the stressfulness scale from the Stress Appraisal

20 Measure (Peacock \& Wong, 1990). Peacock and Wong demonstrated that the stressfulness scale

21 had good internal consistency and was related to mood and psychological distress. The scale

22 consists of four questions, which were reworded for this study to focus on a competition.

23 Sample items included "Does this competition tax or exceed my coping resources?" and "To 
1 what extent do I perceive this competition as stressful?" Participants responded on a five-point

2 scale ranging from 0 (not at all) to 4 (extremely). The reflective latent variable score was

3 calculated and higher scores reflected higher levels of stress. The coefficient alpha reliability

4 was .81 in the present study.

5 Self-Confidence. Self-confidence was assessed using the five-item scale from the

6 revised Competitive State Anxiety Inventory-2, which Cox, Martens, and Russell (2003) found

7 had good internal consistency and construct validity. Participants rated how confident they felt

8 about their upcoming competition on a four-point scale ranging from 0 (not at all) to 3 (very

9 much so). The reflective latent variable score was calculated and higher scores reflected higher

10 levels of self-confidence. The coefficient alpha reliability was .92 at both time points.

11 Affect. Positive and negative affect were assessed using the Positive and Negative Affect

12 Schedule (Watson, Clark, \& Tellegen, 1988). Watson et al. demonstrated the positive and

13 negative affect subscales both had good internal consistency and were correlated with other

14 mood-related measures. Participants reported how they currently felt about the upcoming

15 competition by rating the 20 adjectives on a five-point scale ranging from 0 (not at all) to 4

16 (extremely). Reflective latent variable scores were calculated for positive and negative affect.

17 Higher scores reflected higher positive and negative affect respectively. The coefficient alpha

18 reliabilities were $.88-.91$ across the two time points.

19 Analyses

20 All analyses were conducted in WarpPLS 3.0. We first examined the four-dimensional,

21 unidimensional, and higher-order measurement models. We then examined the main and stress-

22 buffering effects of the four ARSQ dimensions on time 2 self-confidence, positive affect, and

23 negative affect, controlling for time 1 levels of that outcome variable. For each outcome 
1 variable, we examined a path model with direct paths to the time 2 outcome from the time 1 level

2 of that variable, stress, and the four ARSQ dimensions, and moderating paths from each ARSQ

3 dimension to the path between stress and the time 2 outcome. The models were then retested

4 using a unidimensional received support score instead of the four dimensions, and then with a

5 higher-order received support score.

6 A feature of WarpPLS 3.0 is that it searches for nonlinear relationships automatically and

7 determines if paths are best represented as linear or nonlinear. We examined the magnitude,

8 statistical significance, and effect size (Cohen's $1988 f$-squared coefficients) of each standardized

9 path coefficient, and whether the paths were linear or nonlinear. In a nonlinear path, the

10 coefficient reported represents the overall linear trend of that relationship (Kock, 2012). An

11 alpha level of .05 was used for all statistical tests.

\section{Results and Discussion}

\section{ARSQ Measurement Model}

14 The results largely replicated those found in Studies 2 and 3. For the four-dimensional

15 model, all indicator weights were significant (see Table 1) and the mean of the indicator VIFs

16 was 2.75 (range 1.70-4.04). The correlations between the four dimensions were $r \mathrm{~s}=.62-85(p \mathrm{~s}<$

17.001 ) and the mean of the full collinearity VIFs for the four dimensions was 4.48 .

18 The unidimensional and higher-order models were also good. For the unidimensional

19 model, all indicator weights were significant $(b \mathrm{~s}=.05-.06, S E \mathrm{~s}=.01-.02, p \mathrm{~s}<.001)$ and the

20 mean of the indicator VIFs was 3.49 (range 2.37-5.46). For the higher-order model, all four

21 indicator weights to the higher-order construct were significant $(b \mathrm{~s}=.27-.29, S E \mathrm{~s}=.01-.02, p \mathrm{~s}<$

22.001 ) and the mean of the VIFs of the four higher-order indicators was 4.48. Overall these

23 findings provide further evidence, with a third sample, that the dimensional structure of the 
1 ARSQ could be conceptualized in different ways.

\section{Relationships with Self-Confidence and Affect}

3 Descriptive statistics and correlations between variables are displayed in Table 2. With

4 the exception of time 1 positive affect and esteem support, the initial levels of the outcome

5 variables were not significantly correlated with the ARSQ dimensions. In contrast, with the

6 exception of emotional support and time 2 negative affect, the correlations between the ARSQ

7 dimensions and time 2 outcomes were all significant. Given the ARSQ was completed at time 2,

8 the correlations suggest that received support was more strongly related to current levels of self-

9 confidence and affect than to previous levels.

10 The path analyses found that when the initial levels of the outcome variable and stress

11 were controlled for, emotional and esteem support both significantly predicted time 2 self-

12 confidence and positive affect. The relationship between emotional support and self-confidence

13 was linear, whereas the other relationships were nonlinear. The relationships of emotional and

14 esteem support with positive affect became stronger as levels of support increased. The

15 relationship between esteem support and self-confidence reflected a slight S-curve; the

16 relationship was strongest at moderate levels of esteem support (within 1.5 SDs of $M$ ), and

17 weaker at more extreme levels of esteem support. The ARSQ dimensions did not moderate the

18 paths from stress to self-confidence or positive affect. The inclusion of moderating paths,

19 however, did lead to collinearity concerns, with some high indicator VIFs $(M=5.30$, range 1.38 -

20 13.29). Given the potential for collinearity concerns, Kock (2012) suggested moderating paths

21 should be included sparingly in models. We, therefore, reran the self-confidence and positive

22 affect models with all moderating links removed. The mean of the indicator VIFs was reduced

23 to 2.61 and the pattern of significant relationships remained unaltered (see Table 3). The effect 
1 sizes indicated the importance of esteem support for both self-confidence and positive affect

2 (medium-large effects; Cohen, 1988). Previous literature in both social (Cutrona \& Russell,

3 1990) and sport psychology (Rees et al., 2007) has also noted the importance of esteem support

4 in achievement contexts.

5 In contrast to the findings for self-confidence and positive affect, the ARSQ dimensions

6 did not significantly predict time 2 negative affect when included alongside time 1 negative

7 affect and stress. Tangible support, however, did significantly moderate the relationship between

8 stress and time 2 negative affect. Following high VIFs due to the product term indicators, we

9 reran the model with the moderating paths from emotional, esteem, and informational support

10 removed. The mean of the indicator VIFs was reduced to 4.35 and the pattern of results

11 remained unaltered (see Table 3). The negative coefficient of the significant moderating path

12 was generally consistent with a stress-buffering explanation: the relationship between stress and

13 negative affect was weaker at high levels of tangible support compared to low levels of tangible

14 support. To further explore the nature of the moderating link, we examined the graph (see Figure

15 1), which depicts the relationship between stress and negative affect at low (below Mdn) and

16 high (above $M d n$ ) levels of tangible support. The graph suggests that at moderate levels of stress

17 (within 1.5 SDs of $M$ ), individuals with low tangible support reported similar negative affect to

18 those with high tangible support. At high levels of stress, however, individuals with low tangible

19 support reported greater negative affect than individuals with high tangible support. In contrast,

20 at very low levels of stress individuals with low tangible support actually reported less negative

21 affect than those with high tangible support. This finding highlights a potentially complex

22 relationship between tangible support and negative affect, and that receiving practical assistance

23 is not always beneficial. In social psychology, Finch et al. (1997) found that tangible assistance 
1 was positively related to depression. The receipt of tangible support may be helpful when

2 athletes are under stress, but when there is minimal stress tangible support may be seen as

3 controlling, unhelpful, or communicating a sense of inefficacy to the athlete, which may increase

4 negative affect.

5 Substituting the unidimensional received support score and then the higher-order received

6 support construct in place of the four ARSQ dimensions found the same overall pattern of

7 results. Individuals who reported receiving more support also reported higher self-confidence

$8(b \mathrm{~s}=.35, S E \mathrm{~s}=.05, p \mathrm{~s}<.001)$ and positive affect $(b \mathrm{~s}=.40, S E \mathrm{~s}=.06, p \mathrm{~s}<.001)$, and lower

9 negative affect $(b \mathrm{~s}=-.08, S E \mathrm{~s}=.05, p \mathrm{~s}=.04)$. The unidimensional and higher-order constructs

10 also both significantly moderated the relationship between stress and negative affect $(b \mathrm{~s}=-.12$,

$11 S E \mathrm{~s}=.06, p \mathrm{~s}=.02)$. The graphs of these interactions were congruent with that of Figure 1.

12 The above findings provide evidence for the nomological validity of the ARSQ and

13 suggest that the received support dimensions may have different relationships with outcomes.

14 These findings extend our understanding into the effects of received support in sport. For

15 example, Rees and Freeman (2007) had previously demonstrated that received support was

16 associated with main and stress-buffering effects on self-confidence, but their measure did not

17 distinguish between support dimensions. The present findings highlight the benefit of using

18 multidimensional measures of support, as the differential relationships support dimensions have

19 with outcomes can be elucidated.

\section{General Discussion}

21 Researchers have argued that the creation of theoretically-based measures specific to

22 sport are required to develop understanding of the effects of received support in sport (Bianco \&

23 Eklund, 2001; Holt \& Hoar, 2006; Rees, 2007). The purpose of the present article, therefore, 
1 was to develop a measure of received support specific to sport. Four studies provided evidence

2 for construct validity of the ARSQ, which was generally consistent with our predictions. Study 1

3 provided evidence for the content validity of the ARSQ. Using a formative measurement

4 approach, Study 2 then provided initial evidence of the dimensional structure, which was further

5 confirmed with independent samples in Studies 3 and 4. Study 3 also provided partial evidence

6 for convergent validity of the ARSQ, and Study 4 provided evidence of its nomological validity.

7 The findings highlighted that the ARSQ could be used to form a unidimensonal or higher-order

8 received support construct, but the indicator VIFs were consistently lower within the four-

9 dimensional model and there is potential theoretical and applied insight to be gained by

10 recognizing different dimensions of received support. The studies also demonstrated the

11 potential utility for sport psychologists of partial least squares structural equation modeling,

12 formative measurement models, and the use of WarpPLS.

13 Sarason and Sarason (2009) noted that the limited conceptual foundation of many social

14 support questionnaires has impeded research efforts. Consistent with our hypotheses, and

15 previous research in both sport (e.g., Rees \& Hardy, 2000) and social psychology (e.g., Cutrona

$16 \&$ Russell, 1990), we found support for a four-dimensional structure of the ARSQ reflecting

17 emotional, esteem, informational, and tangible support. The ability to replicate this finding

18 across three samples of athletes strengthens our confidence in this dimensional structure and the

19 measure. Similar to other social support measures such as the ISSB, PASS-Q, and Support in

20 Intimate Relationships Rating Scale (Barry et al., 2009), however, the correlations between the

21 ARSQ dimensions were moderate to high. Despite the conceptual distinction between

22 dimensions, correlations may be significant because athletes are often given multiple forms of

23 help and support (Bianco, 2001). The correlations could also reflect the existence of a 
1 unidimensional or higher-order received support construct. Both of these models were found to

2 be good in Studies 2-4, which offers promising flexibility for researchers, depending on the goals

3 of their studies. Similarly, in examining the factor structure of the ISSB, Finch et al. (1997)

4 found that although a four-dimensional model had a superior model fit, the fit of a

5 unidimensional model was also reasonable. When using the ARSQ, researchers could

6 legitimately focus on overall received support, which would reduce model complexity and offer

7 a more parsimonious approach when examining the effects of received support in sporting

8 contexts. In contrast, the VIFs provided evidence that the dimensions were sufficiently distinct

9 from each other to be considered unique forms of support. We argue that examining different

10 dimensions of support is generally preferable as identifying which forms of support are most

11 beneficial may help provide a greater focus when designing received support interventions.

12 In addition to factorial validity, evidence was provided for the additional psychometric

13 properties of the ARSQ. In particular, partial evidence of convergent validity was provided by

14 the moderate to strong correlations between the respective dimensions of the ARSQ and ISSB.

15 Although the correlations between non-corresponding ARSQ and ISSB dimensions were also

16 significant, a $\chi^{2}$ analysis demonstrated that the proportion of these correlations that were below

17 the mean of the four hypothesized correlations was significantly different than might be expected

18 due to chance. Finally, similar to existing social support measures, such as the ISSB and PASS-

19 Q, the ARSQ dimensions had weak correlations with negative affectivity and social desirability.

20 Previous research has made a valuable contribution in highlighting the potential positive

21 role of received support in sport. Studies, however, have generally used received support

22 measures that have focused on global perceptions by using overall impression responses such as

23 'a lot' (Rees \& Freeman, 2007) or 'very much' (Zourbanos et al., 2011). In contrast, the ARSQ 
1 employed a frequency style response similar to the ISSB. Researchers have argued that asking

2 participants to recall the frequency of specific behaviors might more accurately reflect the actual

3 support received (Haber et al., 2007). Further, researchers have often developed unique

4 measures for their studies (e.g., Freeman \& Rees, 2008; Zourbanos et al., 2011). The ability to

5 compare findings and establish strong conclusions about the effects of received support could be

6 hindered by the employment of multiple novel measures (Holt \& Hoar, 2006). The development

7 and consistent use of the ARSQ can facilitate attempts to synthesize research findings and

8 address theoretically interesting questions, such as which dimensions of received support are

9 most beneficial and under what conditions? The results of Study 4 provided initial answers to

10 these questions, as well as demonstrating the nomological validity of the ARSQ.

11 The effects of different dimensions of received support have rarely been examined with

12 quantitative approaches in sport. For example, although received support has been linked to self-

13 confidence (Rees \& Freeman, 2007) and performance (Freeman \& Rees, 2008), these studies

14 used aggregate measures of received support rather than distinguishing between dimensions.

15 Such approaches obscure any differential relationships that support dimensions have with

16 outcomes. In social psychology unique relationships for received support dimensions have been

17 noted with marital adjustment (Barry et al., 2009), life satisfaction (Finch et al., 1997), and well-

18 being (Reinhardt et al., 2006). For example, Finch et al. (1997) found that when the four ISSB

19 dimensions were considered simultaneously only positive social exchange (similar to esteem

20 support) made a significant unique contribution to the prediction of life satisfaction, despite all

21 dimensions and an aggregate score having significant bivariate correlations with life satisfaction.

22 Similarly, although all ARSQ dimensions had significant bivariate correlations with both self-

23 confidence and positive affect in Study 4, when the dimensions were considered simultaneously 
1 only emotional and esteem support significantly predicted these outcomes. These findings

2 suggest that receiving higher levels of emotional and esteem support were associated with higher

3 levels of self-confidence and positive affect, although the effect sizes indicated the particular

4 importance of esteem support. Research that examines the mechanisms through which received

5 esteem support operates is required, but athletes who receive frequent encouragement and

6 positive reinforcement irrespective of stress may experience boosts to their sense of control,

7 mastery, and self-esteem, leading to higher self-confidence and positive affect.

8 Study 4 found that tangible support moderated the relationship between stress and

9 negative affect. This moderating effect was generally consistent with the stress-buffering model:

10 the relationship between stress and negative affect was weaker at high levels of tangible support

11 compared to low levels of tangible support. As such, to maintain low levels of negative affect,

12 athletes under high levels of stress may benefit from receiving tangible support, including help

13 with tasks and planning their training. In contrast, under low levels of stress, receiving high

14 levels of tangible support was associated with higher negative affect compared to low tangible

15 support. This reinforces the notion that not all forms of support are universally beneficial (Finch

16 et al., 1997; Reinhardt et al., 2006; Udry et al., 1997). Bianco and Eklund (2001) proposed that

17 received support is primarily associated with stress-buffering effects. In contrast, the present

18 findings are consistent with the position that received support can exert both main and stress-

19 buffering effects (Cohen et al., 2000), but at times it is also unrelated to outcomes or even linked

20 to detrimental effects (for a review, see Lakey \& Orehek, 2011). The findings suggest that

21 whether received support is beneficial may depend on both the dimension of support and the

22 outcome of interest. Further, the findings highlight the insight gained by employing nonlinear

23 analyses, which can offer a more nuanced understanding of the complex relationships between 
1 received support dimensions and outcomes.

2 Despite being frequently used in organizational psychology, formative models have

3 rarely been adopted in either social support or sport psychology research. We would encourage

4 researchers to consider whether formative models may be appropriate in some contexts. Indeed,

5 Gottlieb and Bergen (2010) have recently highlighted that reflective indices of reliability (e.g.,

6 internal consistency) might not be appropriate for some received support measures, such as the

7 ISSB, because the receipt of one supportive behavior does not necessarily mean other behaviors

8 will also be received. Constructs, however, are not inherently formative or reflective, so it is

9 important to articulate why a given approach has been favored (Diamantopoulos \& Winklhofer,

10 2001; MacKenzie et al., 2011). The decision is not without importance because implementing

11 reflective versus formative analyses can lead to divergent results in the development of a

12 questionnaire (Diamantopoulos \& Siguaw, 2006; MacKenzie, Podsakoff, \& Jarvis, 2005) ${ }^{1}$. As

13 Diamantopoulos and Siguaw (2006) noted, reflective approaches focus on the covariance

14 between items and emphasize unidimensionality and internal consistency within a subscale,

15 whereas formative approaches focus on reducing multicollinearity between items.

16 Some limitations of the present studies should be noted. First, the high correlations

17 between ARSQ dimensions may raise some concerns over the discriminant validity of the

18 subscales. Similarly, the good fit of the unidimensional and higher-order models may raise

19 questions as to the multidimensional nature of support. However, as discussed above, there was

20 support for a four-dimensional model in all studies and the dimensions did have different

21 relationships with outcomes in Study 4. Second, the mean age and ethnic diversity of

22 participants was similar across all studies, so caution should be exerted over generalizing the

23 findings to other samples. Third, the different time period used in the ARSQ (one week) and 
1 ISSB (one month) may have attenuated the correlations between the measures in Study 3.

2 Fourth, the design of Study 4 precludes causal inferences. Participants with higher levels of self-

3 confidence and positive affect at time 2 may have reported receiving more support, rather than

4 support leading to more favorable outcomes. Finally, the ARSQ did not include filter questions

5 to permit respondents to highlight items that were not applicable to them. For example, in their

6 off-season, athletes may have less need for some tangible support such as help at competitions.

$7 \quad$ Item response theory could be useful in exploring filter questions in future research (Reardon \&

8 Raudenbush, 2006).

9 In both Study 4 and previous research (e.g., Reinhardt et al., 2006), not all received

10 support dimensions were associated with beneficial effects on all outcomes. Future research

11 could use the ARSQ to examine factors that moderate the effectiveness of received support,

12 including task-, recipient-, or provider-related factors (Uchino, Carlisle, Birmingham, \& Vaughn,

13 2011). For example, the effectiveness of received support may depend on the match between the

14 dimension of support and the needs arising in a specific situation, the timing of support, whether

15 athletes want support, or who is providing the support. Future research could also consider the

16 distinction between the quantity of received support and athletes' satisfaction with that support.

17 In health psychology, Fiorillo and Sabatini (2011) found that satisfaction with, rather than

18 frequency of, social interactions had a stronger association with individuals' health.

19 In conclusion, the present article has described the development of the ARSQ and

20 provided initial evidence for its construct validity. The development of the ARSQ addressed

21 calls for measures of support that are specific to sport (Bianco \& Eklund, 2001; Holt \& Hoar,

22 2006). Across four studies, there was evidence to support a 22-item measure. The 22 items

23 formed a four-dimensional model reflecting emotional, esteem, informational, and tangible 
1 dimensions, but equally researchers could conceivably adopt a unidimensional or higher-order

2 model if their focus was on overall levels of received support. The four dimensions, however,

3 had different relationships with self-confidence and affect, highlighting the potential insight

4 gained by adopting a multidimensional approach to measuring received support. We hope that

5 the development and consistent use of the ARSQ will facilitate the synthesis of research findings

6 and advance understanding into the impact of received support in sport.

\section{Acknowledgements}

10 The authors thank Miles Peacock, Danielle Howard, Dean Eagles, and Kirsty Edwards for their

11 help during data collection. We also thank three anonymous reviewers for their insightful

12 comments during the review process.

13

14

15

16 


\section{References}

Barrera, M., Jr., Sandler, I. N., \& Ramsay, T. B. (1981). Preliminary development of a scale of social support: Studies on college students. American Journal of Community Psychology, 9, 435-447. doi: 10.1007/BF00918174

Barry, R. A., Bunde, M., Brock, R. L., \& Lawrence, E. (2009). Validity and utility of a multidimensional model of received support in intimate relationships. Journal of Family Psychology, 23, 48-57. doi: 10.1037/a0014174

Bianco, T. (2001). Social support and recovery from sport injury: Elite skiers share their experiences. Research Quarterly For Exercise and Sport, 72, 376-388.

Bianco, T., \& Eklund, R. C. (2001). Conceptual considerations for social support research in sport and exercise settings: The case of sport injury. Journal of Sport \& Exercise Psychology, 23, 85-107.

Carson, F., \& Polman, R. (2012). Experiences of professional rugby union players returning to competition following anterior cruciate ligament reconstruction. Physical Therapy in Sport, 13, 35-40. doi:10.1016/j.ptsp.2010.10.007

Cohen, J. (1988). Statistical power analysis for the behavioral sciences. Hillsdale, NJ: Lawrence Erlbaum.

Cohen, S., Gottlieb, B. H., \& Underwood, L. G. (2000). Social relationships and health. In S. Cohen, L. G. Underwood, \& B. H. Gottlieb (Eds.), Social support measurement and intervention: A guide for health and social scientists (pp. 3-25). New York: Oxford University Press.

Cohen, S., Mermelstein, R., Kamarck, T., \& Hoberman, H. (1985). Measuring the functional components of social support. In I. G. Sarason \& B. R. Sarason (Eds.), Social support: 
Theory, research, and applications (pp.73-94). Dordrecht: Martinus Nijhoff.

2 Cox, R. H., Martens, M. P., \& Russell, W. D. (2003). Measuring anxiety in athletics: The revised Competitive State Anxiety Inventory-2. Journal of Sport \& Exercise Psychology, 25, 519-533.

5

Cutrona, C. E., \& Russell, D. W. (1990). Type of social support and specific stress: Toward a theory of optimal matching. In B. R. Sarason, I. G. Sarason, \& G. R. Pierce (Eds.), Social support: An interactional view (pp. 319-366). New York: Wiley.

Denollet, J. (2005). DS14: Standard assessment of negative affectivity, social inhibition, and type D personality. Psychosomatic Medicine, 67, 89-97. doi: 10.1097/01.psy.0000149256.81953.49

Diamantopoulos, A., \& Winklhofer, H. M. (2001). Index construction with formative indicators: An alternative to scale development. Journal of Marketing Research, 38, 269-277.

Diamantopoulos, A., \& Siguaw, J. A. (2006). Formative versus reflective indicators in organizational measure development: A comparison and empirical illustration. British Journal of Management, 17, 263-282. doi: 10.1111/j.1467-8551.2006.00500.x

Dunn, G. H., Bouffard, M., \& Rogers, W. T. (1999). Assessing item content-relevance in sport psychology scale-construction research: Issues and recommendations. Measurement in Physical Education and Exercise Science, 3, 15-36. doi: 10.1207/s15327841mpee0301_2

Finch, J. F., Barrera, M., Okun, M. A., Bryant, W. H. M., Pool, G. J., \& Snow-Turek, A. L. (1997). The factor structure of received social support: Dimensionality and the prediction of depression and life satisfaction. Journal of Social and Clinical Psychology, 16, 323342.

Fiorillo, D., \& Sabatini, F. (2011). Quality and quantity: The role of social interactions in self- 
reported individual health. Social Science \& Medicine, 73, 1644-1652. doi: 10.1016/j.socscimed.2011.09.007

Fletcher, D, \& Sarkar, M. (2012). A grounded theory of psychological resilience in Olympic champions. Psychology of Sport and Exercise, 13, 669-678. doi:10.1016/j.psychsport.2012.04.007

Freeman, P., Coffee, P., \& Rees, T. (2011). The PASS-Q: The perceived available support in sport questionnaire. Journal of Sport \& Exercise Psychology, 33, 54-74.

Freeman, P., \& Rees, T. (2008). The effects of perceived and received support upon objective performance outcome. European Journal of Sport Science, 8, 359-368. doi: $10.1080 / 17461390802261439$

Gottlieb, B. H., \& Bergen, A. E. (2010). Social support concepts and measures. Journal of Psychosomatic Research, 69, 511-520. doi:10.1016/j.jpsychores.2009.10.001

Haber, M., Cohen, J., Lucas, T., \& Baltes, B. (2007). The relationship between self-reported received and perceived social support. American Journal of Community Psychology, 39, 133-144. doi: 10.1007/s10464-007-9100-9

Hair, J. F., Sarstedt, M., Ringle, C. M., \& Mena, J. A. (2012). An assessment of the use of partial least squares structural equation modelling in marketing research. Journal of the Academy of Marketing Science, 40, 414-433. doi: 10.1007/s11747-011-0261-6

Holt, N. L., \& Hoar, S. D. (2006). The multidimensional construct of social support. In S. Hanton \& S. D. Mellalieu (Eds.), Literature reviews in sport psychology (pp. 199-225). Hauppauge, NY: Nova Science.

Kock, N. (2012). WarpPLS 3.0 user manual. Laredo, TX: ScriptWarp Systems.

Kristiansen, E., Murphy, D., \& Roberts, G. C. (2012). Organizational stress and coping in U.S. 
professional soccer. Journal of Applied Sport Psychology, 24, 207-223. doi: $10.1080 / 10413200.2011 .614319$

Lakey, B., \& Orehek, E. (2011). Relational regulation theory: A new approach to explain the link between perceived social support and mental health. Psychological Review, 118, 482495. doi: $10.1037 / \mathrm{a} 0023477$

Lakey, B., Orehek, E., Hain, K. L., \& VanVleet, M. (2010). Enacted support's links to negative affect and perceived support are more consistent with theory when social influences are isolated from trait influences. Personality and Social Psychology Bulletin, 36, 132-142. doi: $10.1080 / 10413200.2011 .614319$

Lubans, D. R., Morgan, P. J., \& McCormack, A. (2011). Adolescents and school sport: The relationship between beliefs, social support and physical self-perception. Physical Education \& Sport Pedagogy, 16, 237-250. doi: 10.1080/17408989.2010.532784

MacKenzie, S. B., Podsakoff, P. M., \& Jarvis, C. B. (2005). The problem of measurement model misspecification in behavioral and organizational research and some recommended solutions. Journal of Applied Psychology, 90, 710-730. doi: 10.1037/0021-9010.90.4.710

MacKenzie, S. B., Podsakoff, P. M., \& Podsakoff, N. P. (2011). Construct measurement and validation procedures in MIS and behavioural research: Integrating new and existing techniques. MIS Quarterly, 35, 293-334.

Peacock, E. J., \& Wong, P. T. P. (1990). The Stress Appraisal Measure (SAM): A multidimensional approach to cognitive appraisal. Stress Medicine, 6, 227-236. doi: $10.1002 / \mathrm{smi} .2460060308$

Polit, D. F., \& Beck, C. T. (2006). The Content Validity Index: Are you sure you know what's being reported? Critique and recommendations. Research in Nursing \& Health, 29, 489- 
497. doi: 10.1002/nur.20147

Raedeke, T. D., \& Smith, A. L. (2004). Coping resources and athlete burnout: An examination of stress mediated and moderation hypotheses. Journal of Sport \& Exercise Psychology, 26, $525-541$.

Reardon, S. F., \& Raudenbush, S. W. (2006). A partial independence item response model for surveys with filter questions. Sociological Methodology, 36, 256-300. doi: $10.1111 / \mathrm{j} .1467-9531.2006 .00181 . x$

Rees, T. (2007). Influence of social support on athletes. In S. Jowett \& D. Lavallee (Eds.), Social psychology in sport (pp. 223-231). Champaign, IL: Human Kinetics.

Rees, T., \& Freeman, P. (2007). The effects of perceived and received support on selfconfidence. Journal of Sports Sciences, 25, 1057-1065. doi: $10.1080 / 02640410600702974$

Rees, T., \& Hardy, L. (2000). An investigation of the social support experiences of high-level sport performers. The Sport Psychologist, 14, 327-347.

Rees, T., Hardy, L., \& Freeman, P. (2007). Stressors, social support and effects upon performance in golf. Journal of Sports Sciences, 25, 33-42. doi: $10.1080 / 02640410600982279$

Rees, T., Ingledew, D. K., \& Hardy, L. (1999). Social support dimensions and components of performance in tennis. Journal of Sports Sciences, 17, 421-429. doi: $10.1080 / 026404199365948$

Reinhardt, J. P., Boerner, K., \& Horowitz, A. (2006). Good to have but not to use: Differential impact of perceived and received support on well-being. Journal of Social and Personal Relationships, 23, 117-129. doi: 10.1177/0265407506060182 
1 Reynolds, W. M. (1982). Development of reliable and valid short forms of the Marlowe-Crowne Social Desirability Scale. Journal of Clinical Psychology, 38, 119-125. doi: $10.1002 / 1097-4679(198201)$

Richman, J. M., Rosenfeld, L. B., \& Hardy, C. J. (1993). The Social Support Survey: A validation of a clinical measure of the social support process. Research on Social Work Practice, 3, 288-311. doi: 10.1177/104973159300300304

Sarason, I. G., Levine, H. M., Basham, R. B., \& Sarason, B. R. (1983). Assessing social support: The Social Support Questionnaire. Journal of Personality and Social Psychology, 44, 127-139. doi: 10.1037/0022-3514.44.1.127

Sarason, I. G., \& Sarason, B. R. (2009). Social support: Mapping the construct. Journal of Social and Personal Relationships, 26, 113-120. doi: 10.1177/0265407509105526

Uchino, B. N., Carlisle, M., Birmingham, W., \& Vaughn, A. A. (2011). Social support and the reactivity hypothesis: Conceptual issues in examining the efficacy of received support during acute psychological stress. Biological Psychology, 86, 137-142. doi:10.1016/j.biopsycho.2010.04.003

Udry, E., Gould, D., Bridges, D., \& Tuffey, S. (1997). People helping people? Examining the social ties of athletes coping with burnout and injury stress. Journal of Sport \& Exercise Psychology, 19, 368-395.

Vangelisti, A. L. (2009). Challenges in conceptualizing social support. Journal of Social and Personal Relationships, 26, 39-51. doi: 10.1177/0265407509105521

Watson, D., Clark, L. A., \& Tellegen, A. (1988). Development and validation of brief measures of positive and negative affect: The PANAS scales. Journal of Personality and Social Psychology, 54, 1063-1070. doi: 10.1037/0022-3514.54.6.1063 
1 Zourbanos, N, Hatzigeorgiadis, A., Goudas, M., Papaioannou, A., Chroni, S., \& Theodorakis, Y. (2011). The social side of self-talk: Relationships between perceptions of support received from the coach and athletes' self-talk. Psychology of Sport and Exercise, 12, 407-414. doi:10.1016/j.psychsport.2011.03.001 
Footnote

$2{ }^{1}$ Adopting a reflective approach with confirmatory factor analysis of the data in Study 2 would

3 lead to the removal of six items. This would result in a good model fit for both a 16-item, four-

4 factor model (Satorra-Bentler $\chi^{2}(98)=178.74, p<.01 ; \mathrm{RMSEA}=.05, p=.33$; $\mathrm{SRMR}=.05$;

$5 \mathrm{CFI}=.96)$ and a higher-order factor model (Satorra-Bentler $\chi^{2}(100)=184.17, p<.01$; RMSEA

$6=.05, p=.30 ; \mathrm{SRMR}=.05 ; \mathrm{CFI}=.96)$. The findings reported throughout this article for

7 convergent and nomological validity would also generally be replicated if a 16-item reflective

8 ARSQ were used instead of the 22-item formative ARSQ. Full details of these alternative

9 analyses can be obtained from the first author.

\section{Figure Caption}

\section{Figure 1.}

23 Interaction of stress and received tangible support upon time 2 negative affect. The relationship 
1 between stress and time 2 negative affect at low (below $M d n$; dashed line) and high (above $M d n$;

2 solid line) levels of received tangible support.

3

4 
Table 1

Indicator weights and standard errors of the 22 item four-dimensional model in Studies 2-4.

\begin{tabular}{|c|c|c|c|c|c|c|}
\hline \multirow{2}{*}{ Dimension and items } & \multicolumn{2}{|c|}{ Study 2} & \multicolumn{2}{|c|}{ Study 3} & \multicolumn{2}{|c|}{ Study 4} \\
\hline & $\beta$ & $S E$ & $\beta$ & $S E$ & $\beta$ & $S E$ \\
\hline \multicolumn{7}{|l|}{ Emotional support } \\
\hline cheer you up & $.23 *$ & .01 & $.22 *$ & .02 & $.22 *$ & .01 \\
\hline listen to you & $.22 *$ & .01 & $.25^{*}$ & .02 & $.22 *$ & .02 \\
\hline show concern for you & $.24 *$ & .01 & $.27 *$ & .01 & $.24^{*}$ & .02 \\
\hline make you feel that they would always be there for you & $.25 *$ & .01 & $.27 *$ & .02 & $.23 *$ & .02 \\
\hline comfort you & $.25 *$ & .01 & $.27 *$ & .02 & $.25^{*}$ & .02 \\
\hline \multicolumn{7}{|l|}{ Esteem support } \\
\hline encourage you & $.21 *$ & .01 & $.21 *$ & .02 & $.21 *$ & .01 \\
\hline emphasize your abilities & $.24 *$ & .01 & $.26^{*}$ & .02 & $.24^{*}$ & .02 \\
\hline tell you, you can do it & $.24^{*}$ & .01 & $.26^{*}$ & .02 & $.24^{*}$ & .02 \\
\hline reinforce the positives & $.24 *$ & .01 & $.27 *$ & .02 & $.24^{*}$ & .01 \\
\hline boost your confidence & $.24 *$ & .01 & $.26^{*}$ & .02 & $.24^{*}$ & .02 \\
\hline \multicolumn{7}{|l|}{ Informational support } \\
\hline give you advice about performing in competitive situations & $.19 *$ & .01 & $.21 *$ & .02 & $.20^{*}$ & .02 \\
\hline give you tactical advice & $.21 *$ & .01 & $.22 *$ & .02 & $.20 *$ & .01 \\
\hline offer you ideas and suggest actions & $.21 *$ & .01 & $.23 *$ & .02 & $.20 *$ & .01 \\
\hline help you put things in perspective & $.20 *$ & .01 & $.23 *$ & .02 & $.20^{*}$ & .02 \\
\hline help you decide what to do & $.20 *$ & .01 & $.23 *$ & .02 & $.20 *$ & .01 \\
\hline give you advice about what to do & $.22 *$ & .01 & $.22 *$ & .03 & $.20 *$ & .01 \\
\hline \multicolumn{7}{|l|}{ Tangible support } \\
\hline help plan your training & $.21 *$ & .01 & $.24 *$ & .02 & $.21 *$ & .01 \\
\hline help with transport to training and competition/matches & $.19 *$ & .01 & $.18 *$ & .03 & $.19 *$ & .01 \\
\hline do things for you at training and competitions/matches & $.21 *$ & .01 & $.24^{*}$ & .02 & $.20 *$ & .01 \\
\hline help set sessions in training & $.22 *$ & .01 & $.27 *$ & .02 & $.21 *$ & .01 \\
\hline help you with tasks & $.18^{*}$ & .01 & $.18^{*}$ & .02 & $.17 *$ & .01 \\
\hline help manage your training sessions & $.22 *$ & .01 & $.27 *$ & .02 & $.21 *$ & .01 \\
\hline
\end{tabular}


Table 2

Descriptive Statistics and Correlations between Variables in Study 4.

\begin{tabular}{|c|c|c|c|c|c|c|c|c|c|c|c|c|}
\hline Variable & $\bar{M}$ & $S D$ & 1 & 2 & 3 & 4 & 5 & 6 & 7 & 8 & 9 & 10 \\
\hline 1. Time 1 Self-Confidence & 1.88 & .71 & & & & & & & & & & \\
\hline 2. Time 1 Positive Affect & 2.10 & .79 & $.50 *$ & & & & & & & & & \\
\hline 3. Time 1 Negative Affect & .50 & .54 & $-.14^{*}$ & .11 & & & & & & & & \\
\hline 4. Stress & 1.49 & .79 & $-.27^{*}$ & -.01 & $.47^{*}$ & & & & & & & \\
\hline 5. Emotional Support & 2.36 & 1.01 & -.01 & .09 & -.03 & .12 & & & & & & \\
\hline 6. Esteem Support & 2.35 & .93 & .06 & $.17 *$ & -.09 & .06 & $.83 *$ & & & & & \\
\hline 7. Informational Support & 2.12 & .90 & .07 & .11 & -.08 & $.17 *$ & $.73 *$ & $.83^{*}$ & & & & \\
\hline 8. Tangible Support & 1.99 & 1.02 & .12 & .03 & -.09 & .11 & $.62 *$ & $.69 *$ & $.85^{*}$ & & & \\
\hline 9. Time 2 Self-Confidence & 2.07 & .77 & $.58^{*}$ & $.41 *$ & $-.30 *$ & $-.14^{*}$ & $.27 *$ & $.43 *$ & $.35 *$ & $.32 *$ & & \\
\hline 10. Time 2 Positive Affect & 2.41 & .78 & $.28^{*}$ & $.50 *$ & -.12 & .06 & $.35^{*}$ & $.53^{*}$ & $.40^{*}$ & $.31 *$ & $.59 *$ & \\
\hline 11. Time 2 Negative Affect & .59 & .59 & $-.23^{*}$ & -.01 & $.68 *$ & $.40^{*}$ & -.03 & $-.16^{*}$ & $-.14^{*}$ & $-.14 *$ & $-.37 *$ & $-.23 *$ \\
\hline
\end{tabular}

Note. $N=219 . *$ denotes correlation significant at .05 level 


\section{THE ARSQ}

Table 3

Results of the Path Models in Study 4: Effects of Time 1 Outcomes, Stress, and Received Su. on Time 2 Self-Confidence and Affect.

\begin{tabular}{llccccc}
\hline Outcome & Independent Variables & $R^{2 \mathrm{a}}$ & $\beta^{\mathrm{b}}$ & $S E^{\mathrm{c}}$ & $p^{\mathrm{d}}$ & $E S^{\mathrm{e}}$ \\
\hline Self-Confidence & Time 1 Self-Confidence & .39 & .56 & .05 & $<.001$ & .34 \\
& Stress & & -.01 & .06 & .41 & .003 \\
& Emotional Support & & .17 & .07 & .01 & .04 \\
& Esteem Support & & .49 & .10 & $<.001$ & .22 \\
& Informational Support & & .06 & .11 & .27 & .02 \\
& Tangible Support & & .04 & .08 & .34 & .01 \\
\hline Positive Affect & Time 1 Positive Affect & .27 & .41 & .06 & $<.001$ & .21 \\
& Stress & & .10 & .09 & .12 & .01 \\
& Emotional Support & & .23 & .11 & .02 & .08 \\
& Esteem Support & & .63 & .12 & $<.001$ & .34 \\
& Informational Support & & .01 & .11 & .46 & .004 \\
& Tangible Support & & .05 & .08 & .27 & .02 \\
\hline Negative Affect & Time 1 Negative Affect & .33 & .58 & .08 & $<.001$ & .39 \\
& Stress & & .07 & .07 & .18 & .03 \\
& Emotional Support & & -.23 & .22 & .16 & .01 \\
& Esteem Support & & -.14 & .12 & .13 & .03 \\
& Informational Support & & -.19 & .19 & .16 & .04 \\
& Tangible Support & & -.01 & .07 & .44 & .002 \\
& Stress x Tangible Support & & -.14 & .06 & .01 & .05 \\
\hline
\end{tabular}

Notes. $N=219 .{ }^{\mathrm{a}} R^{2}$ of overall model. ${ }^{\mathrm{b}}$ Path coefficient in final model. ${ }^{\mathrm{c}} S E$ of $\beta .{ }^{\mathrm{d}}$ Probability

${ }^{\mathrm{e}}$ Effect size: $f$-squared for path coefficient. ${ }^{\mathrm{f}}(\mathrm{L})$ inear or $(\mathrm{W})$ arped path. 